Bangladesh J. Bot. 37(1): 75-80, 2008 (June)

\title{
DISEASE REACTION OF DIFFERENT CROPS AGAINST VIRULENT POTATO ISOLATES OF RHIZOCTONIA SOLANI KUHN
}

\author{
Md. Maniruzzaman Khandaker, Abul Khair ${ }^{1}$, Md. Khurshed Alam Bhuiyan ${ }^{2}$ \\ Department of Botany, Govt. Saadat College, Tangail, Bangladesh
}

Key words: Rhizoctonia solani, Virulent, Pathogenecity, Crop hosts

\begin{abstract}
A total of 33 hosts from different crop types viz. cereal, pulse, oil, vegetable and fiber plants were tested for pathogenicity in artificially inoculated condition against two isolates (BTB115 and DK64) of Rhizoctonia solani, virulent to potato. In respect of symptoms developed on the stems, the most susceptible hosts against both the isolates were string bean, bottle gourd, groundnut, pea and pumpkin. Soybean stems were highly susceptible to DK64 and less susceptible to BTB115. Disease symptoms did not develop on stems of five crops namely brinjal, proso millet (Panicum miliaceum L.), stem-amaranth, kangkong (Ipomoea reptans L.) and tomato. Symptoms appeared on leaves of all the crops tested. Leaves of groundnut, bush bean, bottle gourd and okra were highly susceptible while those of barley and millet were less susceptible.
\end{abstract}

\section{Introduction}

Rhizoctonia solani Kuhn is the imperfect stage of Thanatephorus cucumeris, predominantly a plant pathogenic soil inhabiting fungus (Carling et al. 1990). The fungus occurs worldwide and is very plurivorous, and it is a common pathogen of 250 plant species including commercially grown crops (Mordue 1974, Bolkan 1980). This fungus causes damping off of seedlings, root rot as well as stem canker of growing plants and black scurf of potato tubers (Weinhold and Bowman 1977). The pathogen is multifaceted in nature and attack almost all parts of crop plants. The fungus is troublesome for cabbage, lettuce and sugar beets, but it is particularly destructive to beans, soybeans, pepper and rubber plants causing considerable losses. Through cross pathogenicity of $R$. solani isolates collected from wheat, rice, maize and cotton it was observed that all the four isolates of $R$. solani were pathogenic to rice, maize and wheat but wheat isolates could not infect cotton and isolates from rice and maize had no evident virulence to cotton (Yan et al. 1984). The farmers of Bangladesh grow different crops, at potato fields after harvest of potato and in potato crop season of the following years (Khandaker et al. 2006). The survival of various Rhizoctonia strains may be owing to other crops or weeds acting as secondary hosts. Considerable loss is incurred for potato as well as for other crops grown in potato fields in the following seasons. Present experiment was thus designed to find out the pathogenic reaction of two virulent potato isolates of $R$. solani to other crops, especially the crops which are grown in Bangladesh following harvest of potato.

\section{Materials and Methods}

Two isolates of $R$. solani viz. DK64 and BTB115 which were found to be most virulent to potato were selected during this study for the assessment of disease reaction against 33 hosts from different crop types viz. cereals, pulses, oil, vegetable and fiber plants, which are grown mostly in the potato fields of Bangladesh. Seeds of the crops were collected from Bangladesh Agricultural Research Institute, Gazipur. The experiment was conducted at Bangabandhu Sheikh Mujibur Rahman Agricultural University, Gazipur, Bangladesh.

${ }^{1}$ Department of Botany, Jahangirnagar University, Savar, Dhaka, Bangladesh. ${ }^{2}$ Department of Plant Pathology, Bangabandhu Sheikh Mujibur Rahman Agricultural University, Gazipur, Bangladesh. 
Inoculum of $R$. solani isolates were grown in water soaked wheat grains in flasks and sterilized in autoclave for one hour at $121^{\circ} \mathrm{C}$ and $15 \mathrm{lb}$ pressure on three consecutive days (Balali et al. 1995). Each flask was inoculated with fifteen mycelial plugs, each of $5 \mathrm{~mm}$ diameters, taken from three-day-old culture of each of the isolates grown on PDA medium. The flasks were then incubated at $25^{\circ} \mathrm{C}$ for three weeks. After incubation the colonized wheat grains were air dried on sterilized paper and stored at $4{ }^{\circ} \mathrm{C}$ until use. Pathogenicity of the isolates to hypocotyls of seedlings was tested by growing the plants in soil amended with the test inocula of $R$. solani. The soil used was shifted through a $2 \mathrm{~mm}$ mesh sieve and steam sterilized. For inoculation of the sterilized soil with fungal isolate, $10 \mathrm{~g}$ of inocula was mixed with one $\mathrm{kg}$ of soil and was kept moist for three days. The inoculated soil was filled in polybags $(10 \mathrm{~cm} \times 16 \mathrm{~cm})$ before planting. Inoculation with the two isolates of $R$. solani, DK64 and BTB115, was done separately. Soil in polybags mixed with only autoclaved and air dried wheat grains served as control. Three polybags were used for each treatment and five to ten seeds, based on size, were planted in each polybag. Crop seeds with $90-100 \%$ germination rate were selected for sowing. Thirty days after planting seedlings in each polybag were uprooted and washed under running tap water and rated for disease severity following 0 (no apparent symptoms) to 4 (more than $75 \%$ hypocotyl tissue necrotic or seedling killed) scale as described by Carling and Leiner (1990).

Pathogenicity of the isolates to the leaves of crop hosts was determined by inoculating detached leaves of 30-day-old crops kept in plastic Petri plates (Galindo et al. 1982). Inoculation was made aseptically at the centre of a leaf by placing a $5 \mathrm{~mm}$ mycelial agar disc from a threeday-old culture of each of the two isolates of $R$. solani grown on PDA plates. After inoculation proper moist condition was maintained inside the plastic Petri plates using three-fold filter paper saturated with sterile water. Leaves that received only PDA discs without the test inocula served as control (Fig. 1). There were three leaves per petri dish, replicated three times for each of the crop. Five days after inoculation, the severity of leaf infection was rated following 0 to 4 scales (Bolkan and Ribeiro 1985). Both disease incidence and per cent disease index (PDI) of stems and only PDI of leaves of all the crop hosts were calculated on the basis of arc sine transformed values. PDI of hypocotyls parts (stems) and leaves were measured with the following formula:

$$
\text { PDI }=\frac{\text { Summation of all ratings }}{\text { Number of plants observed } \times \text { maximum rating }(4)} \times 100
$$

List of the 33 host examined for disease reaction is given below:

Cereals: (Barley -Hordium vulgare L., Maize (Zia mays L.), Proso millet (Penicum miliaceum L.), Millet, Italian (Setaria italicum (L.) P.Beauv), Rice (Oryza sativa L.), Sorghum (Sorghum bicolor L. Moench.), Wheat (Triticum aestivum L.); Vegetables: Pac choi (Brassica oleracea L. var. chinensis), String bean (Vigna sequipedalis L.), Bottle gourd (Lagenaria siceraria (Mol.) Standl.), Brinjal (Solanum melongena L.), Tomato (Lycopersicon esculentum Mill.), Cabbage (Brassica oleracea var. capitata), Cauliflower (Brassica oleracea var. botrytis), Chinese cabbage (Brassica chinensis L.), Red amaranth (Amaranthus tricolor L.), Stem amaranth (Amaranthus lividus L.), Kangkong (Ipomoea reptans L.), Okra (Abelmoschus esculentus (L.) Moench.), Chinese cabbage (Brassica chinensis L.), Bush bean (Phaseolus vulgaris L.), Pumpkin (Cucurbita pepo L.); Pulses: Blackgram (Vigna mungo (L.) Hepper.), Chick pea (Cicer aeritinum L.), Lentil (Lens culinaris Medik.), Mung bean (Vigna radiata (L.) R. Wilcz.); Oil crops: Groundnut (Arachis hypogaea L.), Safflower (Carthamus tinctorius L.), Mustard (Brassica juncea (L. ) Czern.), Sunflower (Helianthus annuus L.), Soybean (Glycine max (L.) Merr.); Fibre crops: Kenaf (Hibiscus canabinus L.), Jute (tossa) (Cochorus olitorius L.). 


\section{Results and Discussion}

The disease reaction of the isolates of $R$. solani (DK64 and BTB115) varied considerably among the 33 crop hosts when they were tested under artificial inoculated condition (Table 1 and Fig. 1). No disease symptom was produced on the stems of five crops viz. brinjal, millet, stemamaranth, kangkong and tomato. With DK64 significantly highest disease incidence of stem was recorded on pea, bush bean and bottle gourd followed by safflower, soybean, blackgram, string bean and pumpkin. The range of disease incidence in groundnut, sunflower, mungbean, lentil, chickpea, italian millet, maize, sorghum, okra, cauliflower, pack choi and jute (tossa) were from 40.00 to $66.67 \%$; while those in mustard, rice, wheat, barley, chinese cabbage, cabbage, red amaranth and kenaf were from 13.33 to $36.67 \%$. The highest disease incidence of stems by the $R$. solani isolate BTB115, ranging form 73.33 to $80.00 \%$, were observed in groundnut, bush bean, pea, bottle gourd, pack choi and pumpkin. Significantly lower disease incidence was found in safflower, mungbean, blackgram and string bean (66.67\%); while in lentil, chickpea, maize and kenaf the incidence was $60.00 \%$. Disease incidence of wheat, italian millet, barley, sorghum, okra, red amaranth and jute (tossa) ranged from 33.33 to $53.33 \%$; and the range of disease incidence on stems of sunflower, mustard, soybean, rice, chinese cabbage, cabbage and cauliflower were from 6.67 to $23.33 \%$. The lowest disease incidence $6.67 \%$ on stem was recorded in soybean and

Table 1. Reaction of different crops against two virulent potato isolates of $R$. solani.

\begin{tabular}{|c|c|c|c|c|c|c|}
\hline \multirow[t]{2}{*}{ Host name } & \multicolumn{2}{|c|}{$\begin{array}{c}\text { Disease incidence of stem } \\
(\%)\end{array}$} & \multicolumn{2}{|c|}{ PDI of stem } & \multicolumn{2}{|c|}{ PDI of leaf } \\
\hline & DK64 & ВТВ115 & DK64 & BTB115 & DK64 & BTB115 \\
\hline \multicolumn{7}{|l|}{ Cereals } \\
\hline Barley & $\begin{array}{l}30.00 \mathrm{i}-\mathrm{l} \\
(33.21)\end{array}$ & $\begin{array}{l}40.00 \text { fgh } \\
(39.23)\end{array}$ & $\begin{array}{l}10.83 \text { gh } \\
(19.19)\end{array}$ & $\begin{array}{l}18.33 \mathrm{f}-\mathrm{i} \\
(25.30)\end{array}$ & $\begin{array}{l}6.67 \mathrm{~m} \\
(14.76)\end{array}$ & $\begin{array}{c}13.33 \mathrm{j}-\mathrm{m} \\
(21.15)\end{array}$ \\
\hline Proso millet & $\begin{array}{l}0.00 \mathrm{~m} \\
(1.28)\end{array}$ & $\begin{array}{l}0.00 \mathrm{l} \\
(1.28)\end{array}$ & $\begin{array}{l}0.00 \mathrm{i} \\
(1.28)\end{array}$ & $\begin{array}{l}0.00 \mathrm{l} \\
(1.28)\end{array}$ & $\begin{array}{c}10.00 \mathrm{~lm} \\
(18.44)\end{array}$ & $\begin{array}{l}1.67 \mathrm{n} \\
(5.16)\end{array}$ \\
\hline Millet,Italian & $\begin{array}{l}63.33 \mathrm{~d}-\mathrm{g} \\
(52.77)\end{array}$ & $\begin{array}{l}33.33 \text { ghi } \\
(35.22)\end{array}$ & $\begin{array}{l}40.83 \mathrm{a}-\mathrm{d} \\
(39.72)\end{array}$ & $\begin{array}{l}10.00 \mathrm{hij} \\
(18.44)\end{array}$ & $\begin{array}{l}14.17 \mathrm{jkl} \\
(22.09)\end{array}$ & $\begin{array}{l}5.00 \mathrm{~m} \\
(12.92)\end{array}$ \\
\hline Maize & $\begin{array}{l}60.00 \mathrm{~d}-\mathrm{h} \\
(50.77)\end{array}$ & $\begin{array}{l}60.00 \text { cde } \\
(50.77)\end{array}$ & $\begin{array}{l}33.33 \mathrm{bcd} \\
(34.99)\end{array}$ & $\begin{array}{l}30.00 \mathrm{c}-\mathrm{f} \\
(33.16)\end{array}$ & $\begin{array}{l}25.00 \mathrm{fgh} \\
(29.92)\end{array}$ & $\begin{array}{c}13.33 \mathrm{j}-\mathrm{m} \\
(21.34)\end{array}$ \\
\hline Rice & $\begin{array}{l}13.331 \\
(21.15)\end{array}$ & $\begin{array}{l}26.67 \mathrm{hij} \\
(30.78)\end{array}$ & $\begin{array}{l}5.00 \mathrm{~h} \\
(12.92)\end{array}$ & $\begin{array}{l}6.67 \mathrm{jk} \\
(14.76)\end{array}$ & $\begin{array}{l}26.09 \text { fgh } \\
(30.89)\end{array}$ & $\begin{array}{c}31.67 \mathrm{e}-\mathrm{h} \\
(34.18)\end{array}$ \\
\hline Sorghum & $\begin{array}{l}53.33 \text { e-i } \\
(46.92)\end{array}$ & $\begin{array}{c}53.33 \text { def } \\
(46.92)\end{array}$ & $\begin{array}{l}16.67 \mathrm{efg} \\
(23.85)\end{array}$ & $\begin{array}{l}15.00 \mathrm{~g}-\mathrm{j} \\
(22.60)\end{array}$ & $\begin{array}{l}28.33 \text { fgh } \\
(31.93)\end{array}$ & $\begin{array}{l}23.33 \mathrm{~g}-\mathrm{j} \\
(28.78)\end{array}$ \\
\hline Wheat & $\begin{array}{l}33.33 \mathrm{ijk} \\
(35.21)\end{array}$ & $\begin{array}{l}40.00 \text { fgh } \\
(39.23)\end{array}$ & $\begin{array}{l}10.00 \mathrm{gh} \\
(18.44)\end{array}$ & $\begin{array}{l}11.67 \mathrm{hij} \\
(19.89)\end{array}$ & $\begin{array}{l}23.33 \text { ghi } \\
(28.85)\end{array}$ & $\begin{array}{l}15.00 \mathrm{i}-\mathrm{l} \\
(22.79)\end{array}$ \\
\hline \multicolumn{7}{|l|}{ Vegetables } \\
\hline Pac choi & $\begin{array}{l}53.33 \text { e-i } \\
(46.92)\end{array}$ & $\begin{array}{l}80.00 \mathrm{ab} \\
(63.44)\end{array}$ & $\begin{array}{l}15.00 \mathrm{fgh} \\
(22.60)\end{array}$ & $\begin{array}{c}48.33 \mathrm{ab} \\
(44.03)\end{array}$ & $\begin{array}{c}30.00 \mathrm{fg} \\
(33.21)\end{array}$ & $\begin{array}{c}55.55 \text { bc } \\
(48.19)\end{array}$ \\
\hline String bean & $\begin{array}{c}73.33 \text { b-e } \\
(59.21)\end{array}$ & $\begin{array}{c}66.67 \mathrm{bcd} \\
(54.99)\end{array}$ & $\begin{array}{c}45.00 \mathrm{abc} \\
(42.09)\end{array}$ & $\begin{array}{c}45.00 \mathrm{abc} \\
(42.12)\end{array}$ & $\begin{array}{c}50.00 \text { bc } \\
(45.00)\end{array}$ & $\begin{array}{c}41.66 \mathrm{c}-\mathrm{f} \\
(40.15)\end{array}$ \\
\hline Bottle gourd & $\begin{array}{c}86.67 \mathrm{ab} \\
(71.87)\end{array}$ & $\begin{array}{l}80.00 \mathrm{a} \\
(66.15)\end{array}$ & $\begin{array}{l}51.67 \text { a } \\
(45.97)\end{array}$ & $\begin{array}{l}55.00 \mathrm{a} \\
(47.88)\end{array}$ & $\begin{array}{c}55.55 \mathrm{ab} \\
(48.19)\end{array}$ & $\begin{array}{c}50.00 \mathrm{bc} \\
(45.00)\end{array}$ \\
\hline Brinjal & $\begin{array}{c}0.00 \mathrm{~m} \\
(1.28)\end{array}$ & $\begin{array}{l}0.00 \mathrm{l} \\
(1.28)\end{array}$ & $\begin{array}{l}0.00 \mathrm{i} \\
(1.28)\end{array}$ & $\begin{array}{l}0.00 \mathrm{l} \\
(1.28)\end{array}$ & $\begin{array}{c}10.00 \mathrm{~lm} \\
(18.44)\end{array}$ & $\begin{array}{l}18.33 \mathrm{hi} \\
(25.30)\end{array}$ \\
\hline Bush bean & $\begin{array}{c}80.00 \mathrm{abc} \\
(67.64)\end{array}$ & $\begin{array}{c}73.33 \text { abc } \\
(59.22)\end{array}$ & $\begin{array}{c}33.33 \mathrm{bcd} \\
(34.92)\end{array}$ & $\begin{array}{c}33.33 \text { b-f } \\
(34.99)\end{array}$ & $\begin{array}{l}61.22 \mathrm{a} \\
(51.50)\end{array}$ & $\begin{array}{c}58.38 \mathrm{ab} \\
(49.82)\end{array}$ \\
\hline \multirow[t]{2}{*}{ Cabbage } & $23.33 \mathrm{kl}$ & $23.33 \mathrm{ij}$ & $6.67 \mathrm{~h}$ & $10.00 \mathrm{hij}$ & $25.00 \mathrm{fgh}$ & $27.78 \mathrm{f}-\mathrm{i}$ \\
\hline & (28.07) & (28.78) & $(12.72)$ & $(18.44)$ & $(30.00)$ & (31.78) \\
\hline
\end{tabular}


Table 1 contd.

\begin{tabular}{|c|c|c|c|c|c|c|}
\hline Cauliflower & $\begin{array}{c}40.00 \text { g-k } \\
(38.85)\end{array}$ & $\begin{array}{c}6.67 \mathrm{k} \\
(12.72)\end{array}$ & $\begin{array}{c}16.67 \text { efg } \\
(23.36)\end{array}$ & $\begin{array}{c}1.67 \mathrm{kl} \\
(6.49)\end{array}$ & $\begin{array}{c}11.11 \mathrm{klm} \\
(19.21)\end{array}$ & $\begin{array}{l}8.33 \mathrm{~lm} \\
(16.55)\end{array}$ \\
\hline Chinese cabbage & $\begin{array}{l}23.33 \mathrm{kl} \\
(28.77)\end{array}$ & $\begin{array}{l}16.67 \mathrm{j} \\
(23.85)\end{array}$ & $\begin{array}{l}8.33 \mathrm{gh} \\
(16.60)\end{array}$ & $\begin{array}{l}6.67 \mathrm{jk} \\
(14.76)\end{array}$ & $\begin{array}{l}22.22 \mathrm{ghi} \\
(28.03)\end{array}$ & $\begin{array}{c}23.05 \mathrm{~g}-\mathrm{k} \\
(26.54)\end{array}$ \\
\hline Stem-Amaranth & $\begin{array}{l}0.00 \mathrm{~m} \\
(1.28)\end{array}$ & $\begin{array}{l}0.00 \mathrm{l} \\
(1.28)\end{array}$ & $\begin{array}{l}0.00 \mathrm{i} \\
(1.28)\end{array}$ & $\begin{array}{l}0.00 \mathrm{l} \\
(1.28)\end{array}$ & $\begin{array}{l}8.33 \mathrm{~lm} \\
(16.77)\end{array}$ & $\begin{array}{l}19.44 \mathrm{~h}-\mathrm{l} \\
(25.59)\end{array}$ \\
\hline $\begin{array}{l}\text { Water spinach/ } \\
\text { Kangkong }\end{array}$ & $\begin{array}{c}0.00 \mathrm{~m} \\
(1.28)\end{array}$ & $\begin{array}{l}0.001 \\
(1.28)\end{array}$ & $\begin{array}{l}0.00 \mathrm{i} \\
(1.28)\end{array}$ & $\begin{array}{l}0.00 \mathrm{l} \\
(1.28)\end{array}$ & $\begin{array}{l}22.22 \mathrm{ghi} \\
(28.03)\end{array}$ & $\begin{array}{c}33.33 \mathrm{~d}-\mathrm{g} \\
(35.15)\end{array}$ \\
\hline Red Amaranth & $\begin{array}{c}36.67 \mathrm{~h}-\mathrm{k} \\
(37.22)\end{array}$ & $\begin{array}{l}\text { 33.33 ghi } \\
(35.22)\end{array}$ & $\begin{array}{l}11.67 \text { gh } \\
(19.89)\end{array}$ & $\begin{array}{c}10.83 \mathrm{hij} \\
(19.19)\end{array}$ & $\begin{array}{l}11.11 \mathrm{klm} \\
(19.21)\end{array}$ & $\begin{array}{c}27.78 \mathrm{f}-\mathrm{i} \\
(31.75)\end{array}$ \\
\hline Okra & $\begin{array}{l}60.00 \mathrm{~d}-\mathrm{h} \\
(50.77)\end{array}$ & $\begin{array}{c}46.67 \mathrm{efg} \\
(43.08)\end{array}$ & $\begin{array}{l}28.33 \text { def } \\
(31.31)\end{array}$ & $\begin{array}{l}15.00 \mathrm{hij} \\
(22.60)\end{array}$ & $\begin{array}{l}63.88 \mathrm{a} \\
(53.18)\end{array}$ & $\begin{array}{c}50.00 \mathrm{bc} \\
(45.00)\end{array}$ \\
\hline Pea & $\begin{array}{l}93.33 \mathrm{a} \\
(80.29)\end{array}$ & $\begin{array}{l}80.00 \mathrm{a} \\
(66.15)\end{array}$ & $\begin{array}{c}49.17 \mathrm{ab} \\
(44.52)\end{array}$ & $\begin{array}{c}48.33 \mathrm{ab} \\
(44.03)\end{array}$ & $\begin{array}{c}44.44 \mathrm{~cd} \\
(41.80)\end{array}$ & $\begin{array}{c}44.44 \text { b-e } \\
(41.75)\end{array}$ \\
\hline Pumpkin & $\begin{array}{c}80.00 \mathrm{bcd} \\
(63.44)\end{array}$ & $\begin{array}{c}80.00 \mathrm{ab} \\
(63.44)\end{array}$ & $\begin{array}{l}55.00 \mathrm{a} \\
(47.96)\end{array}$ & $\begin{array}{l}55.00 \mathrm{a} \\
(47.88)\end{array}$ & $\begin{array}{l}23.33 \text { ghi } \\
(28.85)\end{array}$ & $\begin{array}{l}15.00 \mathrm{i}-\mathrm{l} \\
(22.60)\end{array}$ \\
\hline Tomato & $\begin{array}{c}0.00 \mathrm{~m} \\
(1.28)\end{array}$ & $\begin{array}{l}0.001 \\
(1.28)\end{array}$ & $\begin{array}{l}0.00 \mathrm{i} \\
(1.28)\end{array}$ & $\begin{array}{l}0.001 \\
(1.28)\end{array}$ & $\begin{array}{c}33.33 \text { ef } \\
(35.15)\end{array}$ & $\begin{array}{c}25.00 \mathrm{~g}-\mathrm{j} \\
(30.00)\end{array}$ \\
\hline \multicolumn{7}{|l|}{ Pulses } \\
\hline Blackgram & $\begin{array}{l}73.33 \text { b-e } \\
(59.22)\end{array}$ & $\begin{array}{c}66.67 \mathrm{bcd} \\
(54.99)\end{array}$ & $\begin{array}{l}31.67 \mathrm{~cd} \\
(34.15)\end{array}$ & $\begin{array}{c}33.33 \text { b-e } \\
(35.22)\end{array}$ & $\begin{array}{l}10.00 \mathrm{~lm} \\
(18.44)\end{array}$ & $\begin{array}{l}15.00 \mathrm{i}-\mathrm{l} \\
(22.60)\end{array}$ \\
\hline Chick pea & $\begin{array}{c}53.33 \text { e-i } \\
(46.92)\end{array}$ & $\begin{array}{l}60.00 \text { cde } \\
\text { (50.77) }\end{array}$ & $\begin{array}{c}28.33 \text { def } \\
(31.93)\end{array}$ & $\begin{array}{c}28.33 \mathrm{~d}-\mathrm{g} \\
(32.14)\end{array}$ & $\begin{array}{l}25.00 \mathrm{fgh} \\
(29.92)\end{array}$ & $\begin{array}{c}20.00 \text { g-k } \\
(26.56)\end{array}$ \\
\hline Lentil & $\begin{array}{l}66.67 \mathrm{c}-\mathrm{f} \\
(54.99)\end{array}$ & $\begin{array}{l}60.00 \text { cde } \\
(50.77)\end{array}$ & $\begin{array}{c}38.33 \mathrm{a}-\mathrm{d} \\
(38.24)\end{array}$ & $\begin{array}{c}21.67 \mathrm{e}-\mathrm{h} \\
(27.52)\end{array}$ & $\begin{array}{c}23.33 \text { ghi } \\
(28.85)\end{array}$ & $\begin{array}{c}11.67 \mathrm{klm} \\
(19.31)\end{array}$ \\
\hline Mung bean & $\begin{array}{l}66.67 \text { c-f } \\
(54.99)\end{array}$ & $\begin{array}{l}66.67 \mathrm{bcd} \\
(54.99)\end{array}$ & $\begin{array}{l}30.00 \text { cde } \\
(33.00)\end{array}$ & $\begin{array}{c}28.33 \mathrm{~d}-\mathrm{g} \\
(32.01)\end{array}$ & $\begin{array}{l}25.00 \mathrm{fgh} \\
(29.92)\end{array}$ & $\begin{array}{c}28.33 \mathrm{fgh} \\
(32.09)\end{array}$ \\
\hline \multicolumn{7}{|l|}{ Oil crops } \\
\hline Groundnut & $\begin{array}{l}40.00 \text { g-k } \\
(39.23)\end{array}$ & $\begin{array}{c}80.00 \mathrm{ab} \\
(63.44)\end{array}$ & $\begin{array}{l}11.67 \text { gh } \\
(19.89)\end{array}$ & $\begin{array}{c}38.33 \mathrm{a}-\mathrm{d} \\
(40.11)\end{array}$ & $\begin{array}{l}63.88 \mathrm{a} \\
(53.08)\end{array}$ & $\begin{array}{l}69.44 \mathrm{a} \\
(56.60)\end{array}$ \\
\hline Safflower & $\begin{array}{l}73.33 \text { b-e } \\
(59.22)\end{array}$ & $\begin{array}{l}66.67 \mathrm{bcd} \\
(54.99)\end{array}$ & $\begin{array}{l}30.00 \text { cde } \\
(33.00)\end{array}$ & $\begin{array}{c}41.67 \mathrm{a}-\mathrm{d} \\
(40.11)\end{array}$ & $\begin{array}{l}20.00 \mathrm{hij} \\
(26.45)\end{array}$ & $\begin{array}{c}10.00 \mathrm{klm} \\
(18.44)\end{array}$ \\
\hline Mustard & $\begin{array}{l}26.67 \mathrm{jkl} \\
(30.99)\end{array}$ & $\begin{array}{l}23.33 \mathrm{ij} \\
(28.78)\end{array}$ & $\begin{array}{l}8.33 \text { gh } \\
(16.60)\end{array}$ & $\begin{array}{c}8.33 \mathrm{ij} \\
(16.60)\end{array}$ & $\begin{array}{l}10.00 \mathrm{~lm} \\
(18.44)\end{array}$ & $\begin{array}{c}10.00 \mathrm{klm} \\
(18.05)\end{array}$ \\
\hline Sunflower & $\begin{array}{l}40.00 \text { g-k } \\
(38.85)\end{array}$ & $\begin{array}{l}20.00 \mathrm{ij} \\
(26.56)\end{array}$ & $\begin{array}{c}10.00 \mathrm{gh} \\
(18.05)\end{array}$ & $\begin{array}{l}6.67 \mathrm{jk} \\
(14.76)\end{array}$ & $\begin{array}{c}26.67 \mathrm{fgh} \\
(30.99)\end{array}$ & $\begin{array}{l}15.00 \mathrm{i}-\mathrm{l} \\
(22.79)\end{array}$ \\
\hline Soybean & $\begin{array}{c}73.33 \text { b-e } \\
(59.22)\end{array}$ & $\begin{array}{c}6.66 \mathrm{kl} \\
(9.71)\end{array}$ & $\begin{array}{l}43.33 \mathrm{a}-\mathrm{d} \\
(41.12)\end{array}$ & $\begin{array}{l}1.67 \mathrm{l} \\
(5.16)\end{array}$ & $\begin{array}{c}23.33 \text { ghi } \\
(28.85)\end{array}$ & $\begin{array}{c}23.33 \text { g-j } \\
(28.85)\end{array}$ \\
\hline \multicolumn{7}{|l|}{ Fiber crops } \\
\hline Kenaf & $\begin{array}{c}26.67 \mathrm{jkl} \\
(30.78)\end{array}$ & $\begin{array}{c}60.00 \text { cde } \\
(50.77)\end{array}$ & $\begin{array}{c}10.00 \text { gh } \\
(18.05)\end{array}$ & $\begin{array}{c}30.00 \mathrm{c}-\mathrm{f} \\
(33.21)\end{array}$ & $\begin{array}{c}38.88 \text { de } \\
(38.51)\end{array}$ & $\begin{array}{c}47.22 \mathrm{bcd} \\
(43.40)\end{array}$ \\
\hline Jute (tosa) & $\begin{array}{c}46.67 \mathrm{f}-\mathrm{j} \\
(43.07)\end{array}$ & $\begin{array}{c}53.33 \text { def } \\
(46.92)\end{array}$ & $\begin{array}{c}13.33 \text { gh } \\
(21.15)\end{array}$ & $\begin{array}{c}15.00 \mathrm{~g}-\mathrm{j} \\
(22.60)\end{array}$ & $\begin{array}{c}16.67 \mathrm{ijk} \\
(24.05)\end{array}$ & $\begin{array}{l}15.00 \mathrm{i}-\mathrm{l} \\
(22.60)\end{array}$ \\
\hline
\end{tabular}

Figures within parenthesis are arc sine transformed values. Means in a vertical column having common letter(s) do not differ significantly at 5\% level of significance by DMRT

cauliflower. Maximum PDI of stem (55.00\%) due to the isolate DK64 was recorded in pumpkin, which was statistically similar to soybean, lentil, ittalian millet, pea, bottle gourd and string bean followed by maize and bush bean; and ranged from 30.00 - 31.67\% in safflower, mungbean and black gram. Relatively lower PDI (5\% to 28.33\%) were recorded in groundnut, sunflower, mustard, chickpea, rice, wheat, barley, sorghum, okra, chinese cabbage, cabbage, cauliflower, red amaranth, pack choi, kenaf and jute (tosha). Maximum PDI of stems (55.00\%) due to BTB115 
was recorded in bottle gourd and pumpkin, which were similar to groundnut, safflower, pea, bean and pack choi,. The PDI of mungbean, blackgram, lentil, chickpea, maize, bush bean and kenaf ranged from 21.67 to $33.33 \%$. The PDI for sunflower, mustard, soybean, rice, wheat, italian millet, barley, sorghum, okra, chinese cabbage, cabbage, cauliflower, red amaranth, and jute(tosha) varied from 1.67 to $18.33 \%$ (Table 1).
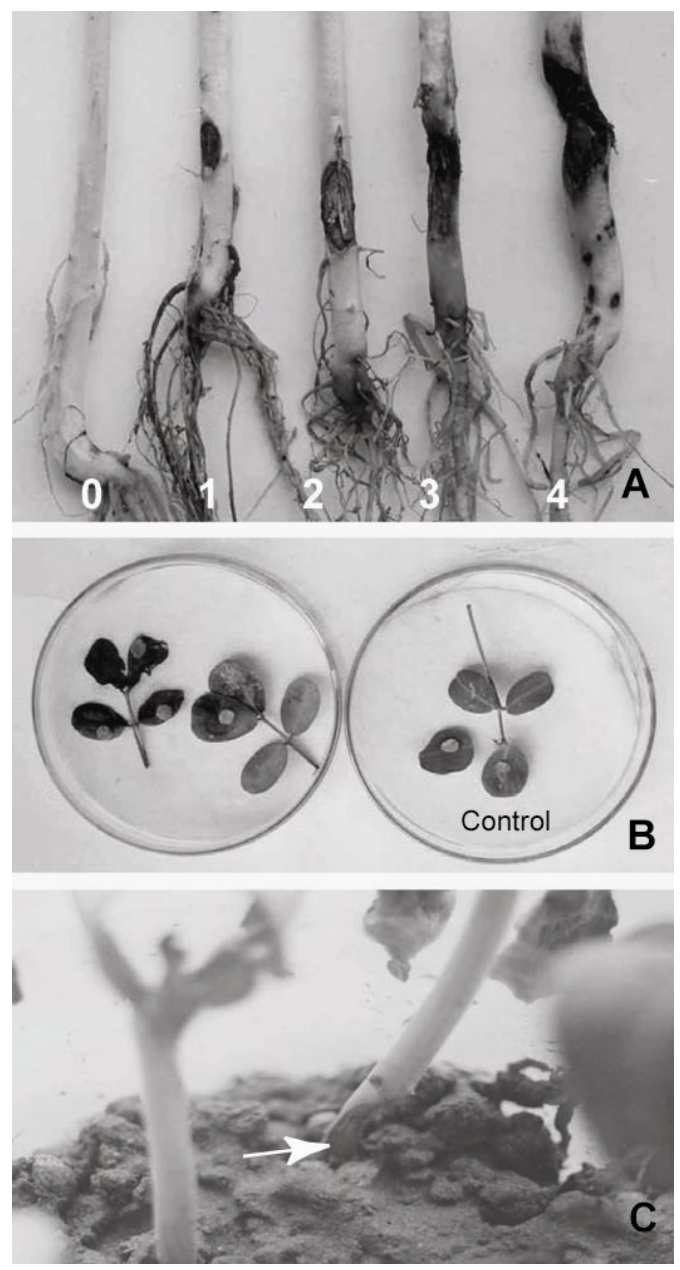

Fig. 1. Rhizoctonia disease symptoms on stems and leaves of crop plants. A. Disease rating from left: 0 (no lesion) to 4 (killed or more than 75\% necrotic lesion) on Bush bean stem, B. Symptoms on groundnut leaf with control plate and C. Bottle gourd stem.

Leaves of all the crops were found to be infected by both the isolates of $R$. solani upon inoculation. Groundnut leaf was found most susceptible against both the isolates where maximum PDI was recorded. Leaves of other crops with higher value of PDI were bush bean, bottle gourd, and okra. The minimum PDI of leaves under DK64 and BTB115 was recorded in barley (6.67\%) and proso millet $(1.67 \%)$ respectively. Trend of pathogenicity of each isolate on stem and leaf of a crop was different. The PDI of stem of groundnut under DK64 was lower but on leaf it was higher, while the isolate BTB115 showed higher PDI on both stem and leaf. 
The disease reaction of the isolates varied considerably among the crops. Only five crops namely brinjal, proso millet, stem amaranth, kangkong and tomato did not respond to $R$. solani isolates in producing disease symptom on stem. Leaves of all the crops were found to be susceptible and produced symptoms when mycelial blocks were inoculated on leaves. Earlier reports indicated that virulent isolate of $R$. solani of a crop host might be virulent or less virulent or avirulent for other crop ( Kang and King 1986, Ogoshi et al. 1990, Nelson et al. 1996, Yang et al. (1996). The above mentioned findings are in close agreement with the present investigation as the pathogenicity of the test isolates varied in different crops, and on stems and leaves of a same crop. In conclusion it may be stated that isolates of $R$. solani which were virulent to potato may survive in the soils and perpetuate with other crop plants grown in the same fields after harvest of potato.

\section{Acknowledgements}

The first author is grateful to the Bangladesh University Grants Commission for granting fellowship and Ministry of Education for providing deputation during the course of this study.

\section{References}

Balali, G.R., S.M. Neate, E.S. Scott, D.L. Whisson and T.J. Wicks. 1995. Anastomosis group and pathogenicity of isolates of Rhizoctonia solani from potato crops in South Australia. Plant Path. 44: 1050-1057.

Baruah, P. and S. Lal. 1981. Host range of Rhizoctonia solani f. sp. sasakii, the incitant of banded sclerotial disease of maize. Indian Phytopath. 34(4): 494-496.

Bolkan, H.A. 1980. Root rots. In: Bean production problems: Disease, insect, soil and climatic constrains of Phaseolus vulgaris. H.F. Schwarts and G.E. Galvez, (Eds), Columbia. 67-100p.

Bolkan, H.A., and R.C.W. Ribeiro. 1985. Anastomosis groups and pathogenicity of Rhizoctonia solani isolates from Brazil. Plant Disease. 69: 599-601.

Carling, D.E. and R.H. Leiner. 1990. Effect of temperature on virulence of Rhizoctonia solani and other Rhizoctonia on potato. Phytopath. 80(10): 930-934.

Carling, D.E., D.J. Helm and R.H. Leiner. 1990. In vitro sensitivity of Rhizoctonia solani and other multinucleate and binucleate Rhizoctonia to selected fungicides. Plant Disease. 74: 860-863.

Galindo, J.J., G.S.Abawi and H.D. Thurston. 1982. Variability among the isolates of Rhizoctonia solani associated with snap bean hypocotyls and soils in New York. Plant Disease. 66: 390-394.

Kang, S.W. and H.K. King. 1986. Incidence and control of bottom rot of chinese cabbage caused by Rhizoctonia solani Kuhn. Korean J. Plant Path. 2(3): 193-198.

Khandaker, M.M., M.K.A. Bhuiyan and A. Khair. 2006. Prevalence of stem canker and black disease of potato in Bangladesh. Bangladesh J. Bot. 35(1): 87-90.

Mordue, J.E.M. 1974. Description of pathogenic fungi. Commonwealth Mycological Institute. pp. 406.

Nelson, B., T. Helms, T. Christianson and I. Kural. 1996. Characterization and pathogenicity of Rhizoctonia from soybean. Plant Disease. 80(1): 74-80.

Ogoshi, A., R.J. Cook and E.N. Bassett. 1990. Rhizoctonia species and anastomosis groups causing root rot of wheat and barley in the Pacific Northwest. Phytopath. 80(9): 785-788.

Weinhold, A.F. and T. Bowman. 1977. Relationship between Rhizoctonia disease of potato and yield. Abst. 69th Annu. Meet. Amer. Phytopath. Soc. p. 119.

Yan, S.Q., B.C. Wu, X.F. Rang, Z.J. Liu and L. Jiang.1984. Sheath blight of cereal crops and the relation between sheath blight of rice, maize and wheat as well as soreshin of cotton. Ecta-PhytopathologicaSinica. 14(1): 25-32.

Yang, J., P.D. Kharbanda, H. Wang and D.W. McAndrew. 1996. Characterization, virulence and genetic variation of Rhizoctonia solani. Plant Disease. 80(5): 513-518. 